\title{
Successful Treatment of Relapsed Chronic Lymphocytic Leukemia with Venetoclax in a Patient with Severe Chronic Kidney Disease
}

Hiroyuki Sugiura $^{1}$, Nobuo Sezaki ${ }^{1}$, Tatsunori Ishikawa ${ }^{1}$, Taiga Kuroi ${ }^{1}$, Sachiyo Okamoto ${ }^{1}$, Naho Nomura ${ }^{1}$, Taro Masunari ${ }^{1}$, Yukio Nakasako ${ }^{1}$, Toru Kiguchi ${ }^{2}$, and Mitsune Tanimoto ${ }^{1}$

${ }^{1}$ Chugoku Chuo Byoin

${ }^{2}$ Dokkyo Medical University Saitama Medical Center

December 23, 2021

\begin{abstract}
Venetoclax is a promising new drug for relapsed or refractory chronic lymphocytic leukemia (CLL). However, venetoclax use had not been reported in severe chronic kidney disease (CKD) patients. We report the first case of relapsed CLL in a severe CKD patient that was successfully treated with venetoclax.
\end{abstract}

\section{Case Report}

Successful Treatment of Relapsed Chronic Lymphocytic Leukemia with Venetoclax in a Patient with Severe Chronic Kidney Disease

Authors: Hiroyuki Sugiura ${ }^{1 *}$, Nobuo Sezaki ${ }^{1}$, Tatsunori Ishikawa ${ }^{1}$, Taiga Kuroi ${ }^{1}$, Sachiyo Okamoto ${ }^{1}$, Naho Nomura ${ }^{1}$, Taro Masunari ${ }^{1}$, Yukio Nakasako ${ }^{2}$, Toru Kiguchi ${ }^{3}$, and Mitsune Tanimoto ${ }^{1}$.

\section{Affiliation:}

${ }^{1}$ Department of Hematology, Chugoku Central Hospital of Japan Mutual Aid Association of Public School Teachers, Fukuyama, Japan

${ }^{2}$ Department of Diabetology and Nephrology, Chugoku Central Hospital of Japan Mutual Aid Association of Public School Teachers, Fukuyama, Japan

${ }^{3}$ Department of Diabetes Endocrinology and Hematology, Dokkyo Medical University Saitama Medical Center, Koshigaya, Japan

*Corresponding author :

Hiroyuki Sugiura M.D., Ph.D.

Department of Hematology, Chugoku Central Hospital of Japan Mutual Aid Association of Public School Teachers

Address: 148-13, Kamiiwanari, Miyuki, Fukuyama, Hiroshima 720-0001, Japan

Phone: +81-84-970-2121

Fax: +81-84-972-8843, E-mail:hiroyuki.sugiura0715@gmail.com

\section{Email Address:}

Hiroyuki Sugiura;hiroyuki.sugiura0715@gmail.com 
Nobuo Sezaki;sezaki-nobuo@kouritu-cch.jp

Tatsunori Ishikawa;t_ishikawa0711@yahoo.co.jp

Taiga Kuroi;blacktiger0119@yahoo.co.jp

Sachiyo Okamoto;sachikado@yahoo.co.jp

Naho Nomura;nnaho0922@gmail.com

Taro Masunari;masunari-tarou@kouritu-cch.jp

Yukio Nakasako;nakasako-yukio@kouritu-cch.jp

Toru Kiguchi;torukiguchi@gmail.com

Mitsune Tanimoto;tanimoto-mitsune@kouritu-cch.jp

\begin{abstract}
Venetoclax is a promising new drug for relapsed or refractory chronic lymphocytic leukemia (CLL). However, venetoclax use had not been reported in severe chronic kidney disease (CKD) patients. We report the first case of relapsed CLL in a severe CKD patient that was successfully treated with venetoclax.
\end{abstract}

\title{
Keywords:
}

chronic lymphocytic leukemia, severe chronic kidney disease, venetoclax, tumor lysis syndrome Introduction

Chronic lymphocytic leukemia (CLL) is a chronic lymphoproliferative disorder characterized by progressive accumulation of functionally incompetent lymphocytes that are usually monoclonal in origin. ${ }^{1}$ Most patients will respond to initial therapy, but disease relapse invariably occurs and becomes refractory with each relapse. Venetoclax, which is an orally bioavailable, selective, small-molecule inhibitor of BCL2, is a new therapeutic agent for relapsed or refractory CLL. ${ }^{2}$ Concerning the toxicity of venetoclax, tumor lysis syndrome (TLS) has been reported as a severe adverse event due to venetoclax treatment and intensive prevention and strict monitoring are recommended according to the risk level of TLS defined by tumor burden and renal function for the use of venetoclax. ${ }^{3}$

Chronic kidney disease (CKD) refers to kidney damage or decreased kidney function for three or more months, regardless of the cause. Decreased kidney function is defined as a glomerular filtration rate (GFR) of less than $60 \mathrm{~mL} / \mathrm{min} / 1.73 \mathrm{~m}^{2}$, and severe CKD (stage 4) is defined as a GFR of less than $30 \mathrm{~mL} / \mathrm{min} / 1.73$ $\mathrm{m}^{2} .{ }^{4}$ To the best of our knowledge, venetoclax use has not been reported in patients with severe CKD. Herein, we describe a case of relapsed CLL patient with severe CKD due to congenital solitary kidney that was successfully treated with venetoclax.

\section{Case Description}

A 78-year-old-man with CKD and congenital solitary kidney was diagnosed with CLL 15 years prior. The Rai classification was intermediate 1, the Binet classification was A, and watchful waiting was adapted. However, lymphocytosis and lymph node enlargement progressed four years after his first visit; therefore, he was prescribed oral fludarabine treatment. Four years after the oral fludarabine treatment, lymph node enlargement progressed again and hence he was prescribed bendamustine with rituximab (BR) therapy. Treatment response was good, but because severe neutropenia occurred, BR therapy was stopped at two cycles. Two years after the BR therapy, CLL progressed again, and BR treatment was administered again for two cycles. Two years after the last BR treatment, lymphocytosis progressed again; therefore, ibrutinib, an inhibitor of Bruton's tyrosine kinase and an effective agent for CLL, was initiated and administered for two years. However, lymphocytosis and lymph node enlargement gradually progressed, and he was admitted to our hospital. 
Laboratory data on the admission day revealed severe lymphocytosis, severely impaired renal function, and mildly increased liver enzymes (Table 1). The serum creatinine level was $2.7 \mathrm{mg} / \mathrm{dL}$, and the estimated GFR from creatinine was $18.7 \mathrm{~mL} / \mathrm{min} / 1.73 \mathrm{~m}^{2}$. He was diagnosed as having a relapse of CLL and complications of severe CKD (stage $4, \mathrm{GFR}<30 \mathrm{~mL} / \mathrm{min}$ ). Computed tomography (CT) revealed systemic lymphadenopathy and splenomegaly. Because of severe lymphocytosis, systemic lymphadenopathy, splenomegaly, and severe CKD from congenital solitary kidney, the risk of TLS was considered extremely high; therefore, oral cyclophosphamide and prednisolone with four cycles of rituximab were administered to reduce tumor burden before venetoclax therapy. The lymphocyte count reduced to 15000 cells $/ \mu \mathrm{L}$, and lymphadenopathy and splenomegaly were ameliorated. After debulking of the tumor, $20 \mathrm{mg} /$ day venetoclax was initiated, along with intensive prevention of TLS involving hydration, $60 \mathrm{mg} /$ day febuxostat, and $7.5 \mathrm{mg} /$ body rasburicase. This is because we assessed that the risk of TLS was still high because of the complications of severe CKD. Strict monitoring of laboratory data at $4,8,12$, and 24 hours after the start of venetoclax (Figure 1) on the first administration day revealed a slight increase in the lactate dehydrogenase level with no laboratory abnormality, which met the criteria for TLS diagnosis. Subsequently, the venetoclax dose was increased weekly to $50 \mathrm{mg}$ /day and $100 \mathrm{mg}$ /day without TLS, but catheter-induced blood stream infection occurred, and venetoclax treatment was stopped for a week. After catheter exchange and antibiotic administration, venetoclax treatment was restarted at a dose of $100 \mathrm{mg} /$ day and increased weekly to $200 \mathrm{mg} /$ day and 400 $\mathrm{mg} /$ day without TLS. Lymphocytosis and soluble interleukin-2 receptor immediately decreased to the normal range. We observed that response to venetoclax treatment was quite good. After venetcoclax reached maintenance dose, he was discharged on day 113 post-admission, and received additional rituximab as outpatient (clinical course during admission is summarized in Figure 2). We told the importance of this case to patient and obtained informed consent to publish.

\section{Discussion}

Venetoclax is an orally bioavailable, selective, small-molecule inhibitor of BCL2 that is highly effective for relapsed or refractory CLL; however, TLS is reported to occur as a severe adverse event of venetoclax. The risk of TLS increases with renal dysfunction, and venetoclax use has not been studied in patients with severe renal impairment. In the phase three study, CLL patients with moderate renal impairment (creatinine clearance $<50 \mathrm{~mL} / \mathrm{min}$ ) were excluded, ${ }^{2}$ and the safety of venetoclax for patients with severe CKD (stage 4 , GFR $<30 \mathrm{~mL} / \mathrm{min}$ ) was not elucidated. To the best of our knowledge, this is the first case in which venetoclax was used for a CLL patient with severe CKD who was not dependent on dialysis. A case report revealed that venetoclax was safely administered in a multiple myeloma patient with renal failure who was dependent on dialysis, ${ }^{5}$ but this patient had undergone dialysis before venetoclax administration. The clinical situation of that case was completely different from that in this case.

Although we considered the dose and schedule about administration of venetoclax to the patient with severe CKD, we decided to administer venetoclax with no dose reduction and no extension of the treatment schedule because venetoclax and its major metabolite are primarily metabolized by the liver cytochrome P450 3A4 enzyme, and urinary clearance is negligible. ${ }^{6}$ In addition, a $50 \%$ dose reduction for patients with severe liver impairment is recommended, ${ }^{7}$ but this patient had only mild liver impairment due to CLL, we assessed that no dose reduction for liver function is needed.

Fortunately, venetoclax was safely administered without the onset of TLS in this case. Debulking by rituximab and cyclophosphamide with prednisolone was successful, and it seemed to reduce TLS risk. In addition, intensive prevention of TLS using hydration, febuxostat, and rasburicase as uric acid-reducing agents seemed to be important too. However, TLS is sometime inevitable and dialysis should be initiated without hesitation if TLS is suspected. Thus, strict monitoring of laboratory data also seemed to be important.

In conclusion, we describe the first case of a relapsed CLL patient with severe CKD due to congenital solitary kidney that was successfully treated with venetoclax. Venetoclax was safely administered by intensive prevention, and strict monitoring of TLS. This report may suggest safety and efficacy about using venetoclax in CLL patients with severe CKD, only if proper risk management of TLS is given. 


\section{Acknowledgments}

We would like to thank all the staff at Chugoku Central Hospital of Japan Mutual Aid Association of Public School Teachers for their contribution to this report.

\section{Authorship contributions}

H.S. managed the clinical practice and authored this case study. N.S. managed and supervised the clinical practice. T.I., T.K., S.O., N.N., T.M., and Y.N. provided advise on the paper. T.K. and M.T. supervised the clinical practice.

\section{Data Availability Statement}

The data during this study available from the corresponding author on reasonable request.

\section{Funding Sources}

No funding was received for this study.

\section{Conflict of interest disclosure}

The authors declare no potential conflicts of interest regarding the publication of this study.

\section{Ethics Approval Statement}

Because all medical care and examination was provided by public health insurance in Japan, this case report was not deemed to be the subject which needed IRB oversight in our institution.

\section{Patient consent Statement}

Written informed consent has been obtained from the patient.

\section{Permission to reproduce material from other sources}

Authors does not borrow any materials from other works.

\section{Clinical trial registration}

The patient did not registrate any clinical trial.

\section{References}

1. World Health Organization Classification of Tumours of Haematopoietic and Lymphoid Tissues. 4th ed. Swerdlow SH, Campo E, Harris NL, et al Eds. IARC Press, Lyon, 2017.

2. Seymour JF, Kipps TJ, Eichhorst B et al. Venetoclax-rituximab in relapsed or refractory chronic lymphocytic leukemia. N Engl J Med 378:1107-1120, 2018.3.

3. Cheson BD, Heitner Enschede S, Cerri E et al. Tumor lysis syndrome in chronic lymphocytic leukemia with novel targeted agents. Oncologist 22:1283-1291, 2017.

4. Levey AS, Eckardt KU, Tsukamoto Y et al. Definition and classification of chronic kidney disease: a position statement from Kidney Disease: Improving Global Outcomes (KDIGO). Kidney Int 67:2089-2100, 2005 .

5. Swan D, Delaney C, Natoni A, O'Dwyer M, Krawczyk J. Successful venetoclax salvage in the setting of refractory, dialysis-dependent multiple myeloma with t(11;14). Hematologica 105:e141-e143, 2020.

6. Salem AH, Agarwal SK, Dunbar M, Enschede SLH, Humerickhouse RA, Wong SL. Pharmacokinetics of Venetoclax, a novel BCL-2 inhibitor, in patients with relapsed or refractory chronic lymphocytic leukemia or non-Hodgkin lymphoma. J Clin Pharmacol 57:484-492, 2017.

7. Salem AH, Dave N, Marbury T et al. Pharmacokinetics of the BCL-2 inhibitor Venetoclax in subjects with hepatic impairment. Clin Pharmacokinet 58:1091-1100, 2019. 


\section{Figure legends}

\section{Figure 1.}

Transition of laboratory data on the first day of administration of venetoclax. LDH and Na follow the left axis, and other markers follow the right axis.

${ }^{*}$ Ca: calcium, IP: inorganic phosphorus, K: potassium, LDH: lactate dehydrogenase, Na: sodium, UA: uric acid

\section{Figure 2.}

Clinical course of the patient from the admission day to the discharge day (day 113). Lymphocyte follows left axis and LDH follows right axis. After debulking by CPA+PSL+Rituximab, venetoclax was started, and the dose was increased gradually; the treatment response was good. Because of the complications of CRBSI, venetoclax treatment was stopped. After the exchange of the catheter and antibiotic therapy, venetoclax was restarted safely.

*Ven: venetoclax, CPA: cyclophosphamide, CRBSI: catheter-related bloodstream infection, PSL: prednisolone, R: rituximab.

Table 1. Laboratory data and BMA findings on admission day

\begin{tabular}{|c|c|}
\hline $\mathrm{CBC}$ and coagulation test & $\mathrm{CBC}$ and coagulatio \\
\hline WBC & $75360 / \mu \mathrm{L}$ \\
\hline Neu & $1 \%$ \\
\hline Lymph & $94 \%$ \\
\hline $\mathrm{RBC}$ & $365 \times 10^{6} / \mu \mathrm{L}$ \\
\hline $\mathrm{Hb}$ & $10.6 \mathrm{~g} / \mathrm{dL}$ \\
\hline Plt & $12 \times 10^{4} / \mu \mathrm{L}$ \\
\hline APTT & $28.5 \mathrm{sec}$ \\
\hline PT-INR & 1.06 \\
\hline Fib & $272 \mathrm{mg} / \mathrm{dL}$ \\
\hline $\mathrm{D}-\mathrm{D}$ & $2.0 \mu \mathrm{g} / \mathrm{mL}$ \\
\hline FDP & $3.1 \mu \mathrm{g} / \mathrm{mL}$ \\
\hline BMA findings & BMA findings \\
\hline NCC $52.3 \times 10^{4} / \mu \mathrm{L}$ & $\mathrm{NCC} 52.3 \times 10^{4} / \mu \mathrm{L}$ \\
\hline Megakaryocyte $24 / \mu \mathrm{L}$ & Megakaryocyte $24 / \mu \mathrm{L}$ \\
\hline Small lymphocyte $94 \%$, CD $5+$, CD20,+ CD23+, light chain $\lambda+$ & Small lymphocyte $94 \%$ \\
\hline Chromosome analysis: 46, XY, del (11) (q?) [16], 46, XY, idem, ?t(2;3) (q21:p13) [3], 46,XY [1] & Chromosome analysis: \\
\hline
\end{tabular}

${ }^{*}$ CBC: complete blood count, WBC: white blood cell, Neu: neutrophil, Lymph: lymphocyte, RBC: red blood cell, Hb: hemoglobin, Plt: platelet, APTT: activated partial thromboplastin time, PT-INR: prothrombin time-international normalized ratio, Fib: fibrinogen, D-D: d-dimer, FDP: fibrin degradation product, BMA: bone marrow aspiration, NCC: nucleated cell count, CD: cluster of differentiation, TP: total protein, Alb: albumin, T-Bil: total bilirubin, AST: aspartate aminotransferase, ALT: alanine aminotransferase, LDH: lactate dehydrogenase, $\gamma$-GTP: $\gamma$-glutamyl transpeptidase, UA: uric acid, Cre: creatinine, BUN: blood urea nitrogen, eGFRcre: estimated glomerular filtration rate from creatine, Na: sodium, K: potassium, Cl: chlorine, Ca: calcium, IP: inorganic phosphorus, sIL-2R: soluble IL-2 receptor. 

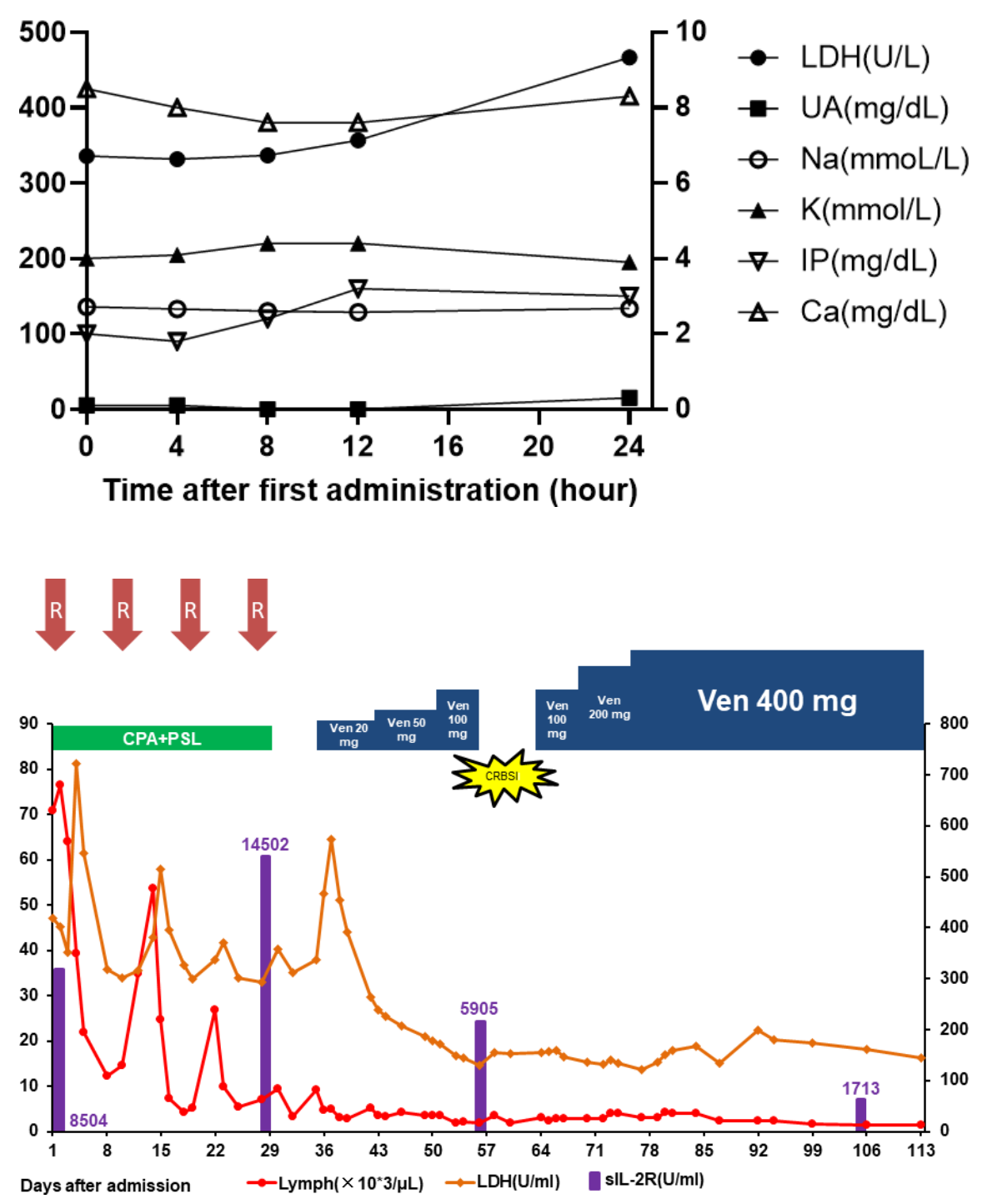